\title{
THE MODULAR SPACE DETERMINED BY A POSITIVE FUNCTION
}

\author{
R. W. BARNARD AND H. H. GOLDSTINE
}

At the suggestion of T. H. Hildebrandt the authors undertook to determine the nature of the space of modular functions of $\mathrm{E}$. $\mathrm{H}$. Moore when the range $\mathfrak{B}$ is taken to be the infinite interval $-\infty$ $<x<+\infty$ and the base matrix $\epsilon$ to be of the form

$$
\epsilon(x, y)=\int_{-\infty}^{+\infty} e^{i(x-y) t} d V(t),
$$

where $V$ is a monotonically increasing bounded function. This form of $\epsilon$ is suggested by the work of Bochner on positive functions. ${ }^{1}$ In this note we determine the form of functions modular as to $\epsilon$ and of the $J$-integral.

To avoid, at first, convergence questions we turn our attention to functions $\phi$ finite as to $\epsilon$, that is, functions of the form

$$
\phi(x)=\sum_{j=1}^{n} \epsilon\left(x, y_{j}\right) a_{j}=\int_{-\infty}^{+\infty} e^{i x t} \lambda(t) d V(t),
$$

where

$$
\lambda(t)=\sum_{j=1}^{n} a_{j} e^{-i y_{j} t} .
$$

In the formulas (2) and (3) the $a_{j}$ are arbitrary constants and the $y_{j}$ are points on the interval $(-\infty,+\infty)$. It is known from standard results in the theory of modular and finite functions ${ }^{2}$ that every function $\phi$ finite as to $\epsilon$ is modular and that

$$
\begin{gathered}
N \phi=J \bar{\phi} \phi=\sum_{i, k=1}^{n} \bar{a}_{j} \epsilon\left(x_{j}, x_{k}\right) a_{k} \\
J \bar{\phi}_{1} \phi_{2}=\left[N\left(\phi_{1}+\phi_{2}\right)-N\left(\phi_{1}-\phi_{2}\right)-i N\left(\phi_{1}+i \phi_{2}\right)\right. \\
\left.+i N\left(\phi_{1}-i \phi_{2}\right)\right] / 4 .
\end{gathered}
$$

Calculating the values of $N \phi$ and $\overline{J \phi}_{1} \phi_{2}$, we see that

$$
J \bar{\phi}_{1} \phi_{2}=\int_{-\infty}^{+\infty} \bar{\lambda}_{1} \lambda_{2} d V, \quad N \phi=\int_{-\infty}^{+\infty}|\lambda|^{2} d V .
$$

Received by the editors March 7, 1942.

${ }^{1} \mathrm{~S}$. Bochner, Monotone Funktionen, Stieltjessche Integrale und harmonische Analyse, Mathematische Annalen, vol. 108 (1933), pp. 378-410.

${ }^{2}$ E. H. Moore, General Analysis, Part II, Philadelphia, 1939, pp. $94 \mathrm{ff}$. 
To determine the form of an arbitrary modular function $\mu$ we consider a sequence

$$
\phi_{n}(x)=\int_{-\infty}^{+\infty} e^{i x t} \lambda_{n} d V
$$

of functions finite as to $\epsilon$, converging mode 2 to a modular function ${ }^{3} \mu$. Since $\phi_{n}$ converges strongly, it follows that

$$
\lim _{m, n} N\left(\phi_{m}-\phi_{n}\right)=\lim _{m, n} \int_{-\infty}^{+\infty}\left|\lambda_{m}-\lambda_{n}\right|^{2} d V=0,
$$

and hence there exists a measurable function $\lambda$ such that $\lambda^{2}$ is integrable with respect to $V$ and ${ }^{4}$

$$
\lim _{n} \int_{-\infty}^{+\infty}\left|\lambda_{n}-\lambda\right|^{2} d V=0
$$

With the help of Schwarz' inequality one sees that

$$
\mu(x)=\int_{-\infty}^{+\infty} e^{i x t} \lambda(t) d V, \quad N \mu=\int_{-\infty}^{+\infty}|\lambda|^{2} d V .
$$

THEOREM 1. To each modular function $\mu$ there corresponds a measurable function $\lambda$ such that $\lambda^{2}$ is integrable with respect to $V$ and

$$
\mu(x)=\int_{-\infty}^{+\infty} e^{i x t} \lambda(t) d V(t) \text { and } N \mu=\int_{-\infty}^{+\infty}|\lambda|^{2} d V .
$$

Conversely, if $\lambda$ is measurable and $\lambda^{2}$ integrable with respect to $V$, then the first of the formulas (5) defines a modular function $\mu$ for which the second of these formulas is valid. If $\mu_{1}, \mu_{2}$ are two modular functions, then

$$
J_{\bar{\mu}_{1} \mu_{2}}=\int_{-\infty}^{+\infty} \bar{\lambda}_{1} \lambda_{2} d V
$$

where $\lambda_{1}, \lambda_{2}$ are the square integrable functions associated with $\mu_{1}, \mu_{2}$.

It remains to prove only the latter part of the theorem. To do this let $\xi(x)=\int_{-\infty}^{+\infty} e^{i x t} \lambda d V$, where $\lambda$ is any measurable function such that $\lambda^{2}$ is integrable with respect to $V$, and let $x_{j}, a_{j}(j=1,2, \cdots, n)$ be constants such that

${ }^{3}$ Ibid., p. 116.

${ }^{4}$ E. W. Hobson, The Theory of Functions of a Real Variable, 2d edition, 1926, vol. II, p. 246. 


$$
\sum_{j, k=1}^{n} \bar{a}_{j} \epsilon\left(x_{j}, x_{k}\right) a_{k}=\int_{-\infty}^{+\infty}\left|\sum_{j=1}^{n} a_{j} e^{-i x_{j} t}\right|^{2} d V \leqq 1 .
$$

It then follows with the help of Schwarz' inequality that

$$
\left|\sum_{j=1}^{n} \bar{a}_{j} \xi\left(x_{j}\right)\right|^{2}=\left|\int_{-\infty}^{+\infty}\left(\sum_{j=1}^{n} \bar{a}_{j} e^{i x_{j} t}\right) \lambda d V\right|^{2} \leqq \int_{-\infty}^{+\infty}|\lambda|^{2} d V,
$$

and hence $\xi$ is modular. ${ }^{5}$ The formula (6) follows at once from the second equation (5) and equation (4).

Finally, we seek conditions that the matrix $\epsilon$ should be proper. These are contained in the following result:

THEOREM 2. The base matrix $\epsilon$ is proper if the measure function $V$ is such that every set $E$ whose complement has zero measure has a finite limit point.

It is clear that

$$
0=\sum_{j, k=1}^{n} \bar{a}_{j} \epsilon\left(x_{j}, x_{k}\right) a_{k}=\int_{-\infty}^{+\infty}\left|\sum_{j=1}^{n} a_{j} e^{-i x_{j} t}\right|^{2} d V
$$

implies the vanishing of the analytic function

$$
\sum_{j=1}^{n} a_{j} e^{-i x_{j} t}
$$

for almost all $t$. If the constants $a_{j}$ were not all zero, the expression (7) would have a non-finite number of zeros in a bounded interval, which is false, and hence $a_{1}, \cdots, a_{n}$ are all zero and $\epsilon$ is a proper matrix.

The University of Chicago and

The University of Michigan

5 Ibid., p. 84. 